\title{
Theoretical and Finite Element Modeling of Fine Kirschner Wires in Ilizarov External Fixator
}

A. R. Zamani

\section{S. 0. Oyadiji}

School of Mechanical, Aerospace and Civil Engineering,

University of Manchester, Manchester M60 1QD, UK
The mechanical behavior of the transosseous elements is a defining factor in the overall stiffness, stability, and reliability of an external fixation system. Mechanics involving the application of thin Kirschner wires in Ilizarov apparatus is yet to be fully explained. To address this problem, load-deflection behavior of the pretensioned thin wires laterally loaded by the bone is necessary to be studied. In this paper, the lateral deflections of thin Kirschner wires are studied both theoretically and computationally. Fully three dimensional finite element (FE) modeling and analyses were performed in which the bone was modeled as a hollow cylinder, and the wire-bone interaction was assumed to be frictionless. The mathematical solution resulted in new exact solutions for the deflection as well as final tension in the wires subjected to the lateral loading under a cylinder representing the bone. Results from the FE analyses turned out to be very close to those from the mathematical solution. The results obtained from theory and FE method are comparable to published experimental findings. Some aspects of the pretensioned thin wire behavior in ring fixation systems, e.g., stiffness-tension proportionality, were revealed in the results. The current study adds to the existing knowledge on the general behavior of tensile elements.

[DOI: $10.1115 / 1.4001815]$

Keywords: Kirschner wire, Ilizarov, pretension, external fixator, finite element method, geometric nonlinearity, contact analysis

\section{Introduction}

Ilizarov appliance is a ring (or circular) external fixation system by which bone fragments are stabilized using thin Kirschner wires (K-wires), which pass through the bone and are fixed to an outer frame of rings and threaded rods [1-4]. It is a modular fixation system, which is assembled around the limb, according to the specific clinical application [3,5], as is illustrated in Fig. 1, and is widely used for treatment of a variety of skeletal deformities and fractures in orthopedics, as well as for limb lengthening and reconstruction procedures [1-3]. The diameter of the wires does not exceed $2 \mathrm{~mm}$, hence the name fine or thin wires. Thicker pins are also used as transosseous elements in Ilizarov assemblies. For the pins, bending can be assumed to be the predominant mode of deformation; thus their mechanical behavior can be described by the existing solutions provided by the beam theories. For the thin wires however, the existing solutions based on the classical beam theory are not applicable. This is due to their slenderness, the application of pretension, and the fact that the tension increases in them after the transverse loading [6,7]. One obvious advantage in utilizing the wires is that their smaller diameter causes minimal invasiveness [8]. Their usage is widely believed to have affected the treatment process positively because they allow interframentary micromotion to occur at the fracture or osteotomy site [4,9-13]. They are also believed to allow conducive stress distribution in metaphyseal treatments [14]. The application of pretension to the wires increases their transverse stiffness considerably, making them an alternative to unilateral pins. Pretensioning and the increase of the tension in the wires due to transverse loading complicate the formulation of their load-deflection behavior, which is the subject of Sec. 2.

The wire-bone interaction is also an important factor vis-à-vis the stiffness and stability of the fixator. There are cases in which a

Manuscript received November 3, 2009; final manuscript received May 14, 2010; published online August 31, 2010. Assoc. Editor: Vijay Goel. small stopper is placed on the K-wires; olive wires are examples of such cases, which are believed to improve stability and overall fixator stiffness of the fixator [4,5,9]. This stabilizing effect of application of olive K-wires demonstrates that, in fact, bone tends to slide along the wires in clinical applications, emphasizing the significance of bone-wire interaction in overall performance of the fixator. Nonetheless, in this paper, the wire is assumed to be smooth, over which the bone is free to slide. Figure 2 illustrates a simple case of a single $\mathrm{K}$-wire over which is pressed down by a cylinder (representing the bone), exerting a load $P$, on the wire vertical to the original axis of the wire. In a previous paper [6], the part of the wire placed under the bone was ignored in a purely tensile formulation. In this paper, however, a tensile model in which the deformation of that part of the K-wire is taken into account is developed. The rest of this paper is attempted at formulation and simulation of the behavior of K-wires assuming ideal conditions of linear elastic material behavior, as well as frictionless interaction of wires and the bone. It is necessary to do so to be able to distinguish the ideal behavior from the effects that are caused by other factors such as slippage of the wires from under the fixation bolts (i.e., clamps), plastic deformation of the wires due to clamping and twisting of the wire [15-18], and so on. It also helps describing the experimental and clinical findings. The assumption of elastic behavior of the $\mathrm{K}$-wires is supported by experimental findings [18].

\section{Mathematical Formulation}

For simplicity, a symmetrical case of a wire being loaded by a cylinder, as illustrated in Fig. 2, is considered for solution, and the wires are modeled mathematically as ideal wires. It means that the deformation is solely attributed to effect of tension in the wire, while any deformations due to bending, shear, or torsion are ignored, hence the name tensile (or wire) model. This means that nonetheless, here the wire is not considered as a chain of separate wire segments of fixed undeformed lengths, but it is being treated 


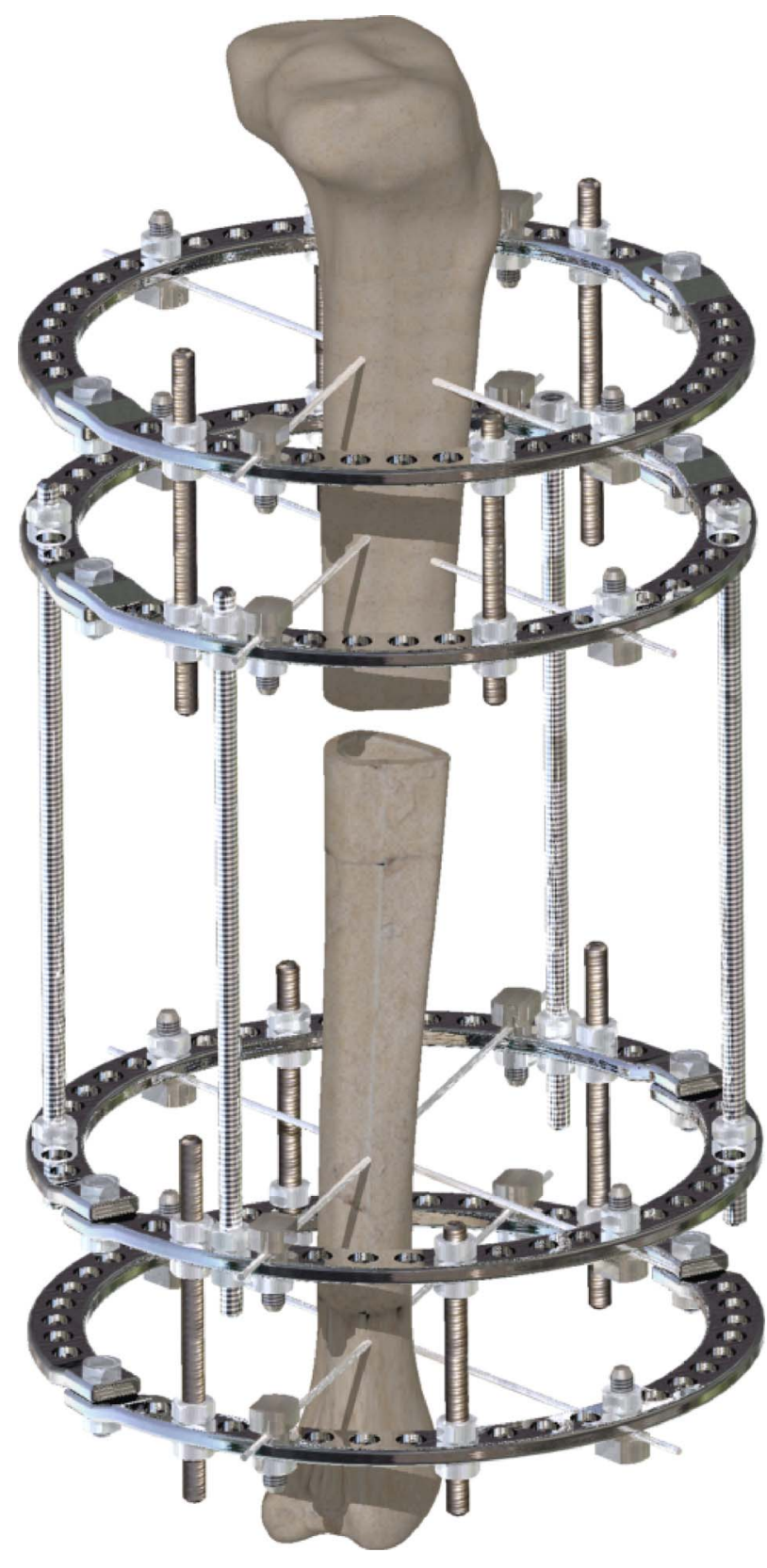

Fig. 1 llizarov ring fixator applied to tibia

as a single wire of a given total undeformed length, which is divided into (three) wire segments due to its deformation. The axial force applied to a wire prior to its fixation on the ring (by the fixation bolts as seen in Fig. 2) is called pretension and is repre-

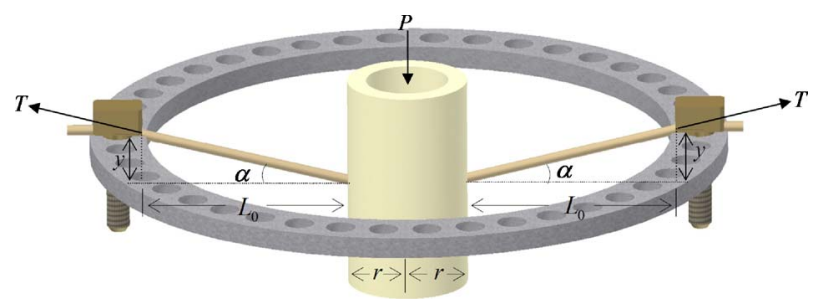

Fig. 2 Transverse deflection of a K-wire passing through a cylinder and fixed to an llizarov full-ring, where cylinder can slide freely over the wire and the wire assumed to be deformed solely due to tension (i.e., no bending in the wire)

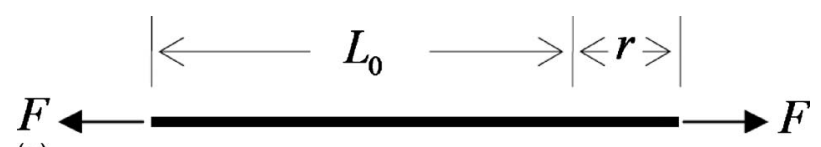

(a)

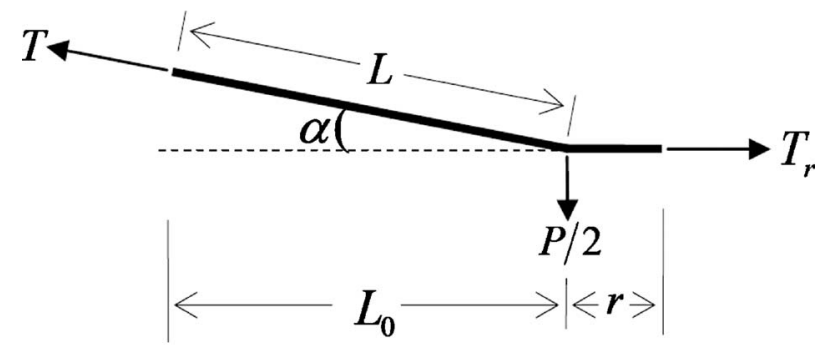

(b)

Fig. 3 Free-body diagram of the K-wire showing the forces acting on half of the wire: (a) after pretensioning and prior to lateral loading and $(b)$ after application of both pretension and lateral load

sented by $F$. The load applied upon the wire by the bone segment (cylinder) vertical to its original axis is referred to as lateral or transverse load and is denoted by $P$.

Each of these forces induces different elongations in each wire segment; therefore, care should to be taken to distinguish between the respective lengths of each wire segment before and after the application of each of these loads. Here the terms unstressed, undeformed, and untensioned are used interchangeably, meaning the length of the wire or wire segment prior to any loading, other terms such as tension-free or stress-free can also be used. The outer radius of the bone at the point of insertion of the wire is called $r$, and the length of the wire segment, which is stretched

due to pretension to cover $r$, is called $r_{0}$ (i.e., $r_{0} \rightarrow r$ ). Thus $r-r_{0}$ is the elongation induced in the wire segment of the length $r_{0}$, due to the pretension force $F$. The bone-clamp distance is denoted by $L_{0}$, which is the length of the wire segment connecting bone to clamp after pretension, as seen in Fig. 3(a), while the untensioned length of the wire segment, which is stretched due to pretensioning force,

$F$, to cover the bone-clamp distance is named $L_{-2}$ (i.e., $L_{-2} \rightarrow L_{0}$ ). Thus the total unstressed length of the wire prior to pretensioning is $L_{-2}+r_{0}$ (of half of total wire span between the clamps as seen in Fig. 3(a)).

As the load $P$ is applied, the tension is increased to the final tension $T$ in the wire in the bone-clamp wire segment. The tension in the wire segment inside the bone is also raised to a new value called $T_{r}$. The length of wire segment, which is stretched due to its $T_{r}$

final tension $\left(T_{r}\right)$ to cover $r$, is called $r_{-1}$ (i.e., $r_{-1} \rightarrow r$ ), and the untensioned length of the wire segment, which is stretched due to the final tension $(T)$ to connect bone to clamp, is called $L_{-1}$ (i.e., $T$

$\left.L_{-1} \rightarrow L\right)$. This means that $L_{-1}+r_{-1}$ also constitutes the total unstressed length (for half of the whole wire span connecting the two clamps). The length of the wire segment connecting bone to clamp after both pretensioning and lateral loading, as seen in Fig. $3(b)$, is called $L$. Static equilibrium of forces in Fig. 3(b) for the horizontal direction gives

$$
T_{r}=T \cos \alpha
$$

and for the vertical direction it gives 


$$
T \sin \alpha=\frac{P}{2}
$$

Assuming linear elasticity for the material behavior, the definitions of $r_{-1}, r_{0}, r, L_{-2}, L_{-1}, L_{0}, F, T$, and $T_{r}$ as mentioned above give

$$
\begin{gathered}
r-r_{-1}=\frac{T_{r} r_{-1}}{E A} \\
r-r_{0}=\frac{F r_{0}}{E A} \\
L_{0}-L_{-2}=\frac{F L_{-2}}{E A}
\end{gathered}
$$

and finally

$$
L-L_{-1}=\frac{T L_{-1}}{E A}
$$

Geometry in Fig. 2 gives

$$
L_{0}=L \cos \alpha
$$

As was said above, the total unstressed length of half of the wire is given by $L_{-2}+r_{0}$ and also by $L_{-1}+r_{-1}$, which are the unstressed lengths before pretensioning and before application of both pretension and lateral load, respectively. Thus

$$
L_{-1}+r_{-1}=L_{-2}+r_{0}
$$

Now there are eight unknowns (namely, $L_{-2}, L_{-1}, L, r_{-1}, r_{0}, T_{r}, T$, and $\alpha$ ) and eight equations, which are Eqs. (1)-(8). Now attempt should be made to solve the equations above simultaneously to obtain a single explicit equation for maximum deflection, namely, $y$. Equation (3) gives

$$
r_{-1}=\left(\frac{E A}{E A+T_{r}}\right) r
$$

Similarly, Eq. (4) gives

$$
r_{0}=\left(\frac{E A}{E A+F}\right) r
$$

Equation (5) gives

$$
L_{0}=\left(\frac{E A+F}{E A}\right) L_{-2}
$$

Finally, Eq. (6) gives

$$
L_{-1}=\left(\frac{E A}{E A+T}\right) L
$$

Equations (9) and (10) give

$$
r_{-1}-r_{0}=E A\left(\frac{1}{E A+T_{r}}-\frac{1}{E A+F}\right) r
$$

Substituting $L_{-2}$ from Eq. (8) into Eq. (11) gives

$$
\left(\frac{E A+F}{E A}\right)\left(L_{-1}+r_{-1}-r_{0}\right)=L_{0}
$$

Substituting $r_{-1}-r_{0}$ from Eq. (13) into Eq. (14) gives

$$
\frac{E A+F}{E A} L_{-1}-\frac{T_{r}-F}{E A+T_{r}} r=L_{0}
$$

Substituting $L_{-1}$ from Eq. (12) into Eq. (15) gives

$$
\frac{E A+F}{E A+T} L-\frac{T_{r}-F}{E A+T_{r}} r=L_{0}
$$

Substituting $T_{r}$ from Eq. (1) into Eq. (16) gives

$$
\frac{E A+F}{E A+T} L-\frac{T \cos \alpha-F}{E A+T \cos \alpha} r=L_{0}
$$

Substituting $L$ from Eq. (7) into Eq. (17)) gives

$$
\left(\frac{E A+F}{E A+T}\right)\left(\frac{L_{0}}{\cos \alpha}\right)+\frac{F-T \cos \alpha}{T \cos \alpha+E A} r=L_{0}
$$

Dividing both sides of the Eq. (18) by $L_{0}$ and referring to $r / L_{0}$ as $j$ give

$$
\left(\frac{E A+F}{E A+T}\right)\left(\frac{1}{\cos \alpha}\right)+\frac{(F-T \cos \alpha)}{T \cos \alpha+E A} j=1
$$

Equation (19) can be rearranged as

$E A(E A+F)+E A(F j-E A) \cos \alpha+T(1+j) \cos \alpha(F-E A \cos \alpha)$

$$
-T^{2}(1+j) \cos ^{2} \alpha=0
$$

Substituting $T$ from Eq. (2) into Eq. (20) gives

$$
\begin{aligned}
& E A(E A+F)+E A(F j-E A) \cos \alpha+\left(\frac{P}{2 \sin \alpha}\right) F(1+j) \cos \alpha \\
& -\left(\frac{P}{2 \sin \alpha}\right) E A(1+j) \cos ^{2} \alpha-\left(\frac{P}{2 \sin \alpha}\right)^{2}(1+j) \cos ^{2} \alpha=0
\end{aligned}
$$

From geometry in Fig. 2, it can be seen that

$$
\cot \alpha=\cos \alpha / \sin \alpha=L_{0} / y
$$

Substituting Eq. (22) into Eq. (21) gives

$$
\begin{aligned}
& E A(E A+F)+E A(F j-E A) \cos \alpha+\left(\frac{P}{2}\right) F(1+j)\left(\frac{L_{0}}{y}\right) \\
& -\left(\frac{P}{2}\right) E A(1+j)\left(\frac{L_{0}}{y}\right) \cos \alpha-\left(\frac{P}{2}\right)^{2}(1+j)\left(\frac{L_{0}}{y}\right)^{2}=0
\end{aligned}
$$

Equation (22) gives

$$
\cos \alpha=\frac{L_{0}}{\sqrt{L_{0}^{2}+y^{2}}}
$$

Substituting $\cos \alpha$ from Eq. (24) into Eq. (23), and rearranging and raising to the second power to eliminate the radical give a sixth degree equation for $y$, which is

$$
a_{6} y^{6}+a_{5} y^{5}+a_{4} y^{4}+a_{3} y^{3}+a_{2} y^{2}+a_{1} y+a_{0}=0
$$

the coefficients of which are

$$
\begin{gathered}
a_{6}=-[4 E A(E A+F)]^{2} \\
a_{5}=-16 E A P F(1+j)(E A+F) L_{0} \\
a_{4}=\left\{(4 E A)^{2}(F j-E A)^{2}-[4 E A(E A+F)]^{2}-[2 P(1+j) F]^{2}\right. \\
\left.+8 E A(E A+F) P^{2}(1+j)\right\} L_{0}^{2} \\
a_{3}=\left\{-(4 E A)^{2}(F j-E A)(1+j) P-16 E A(1+j)(E A+F) P F\right. \\
\left.+4 P^{3} F(1+j)^{2}\right\} L_{0}^{3} \\
a_{2}=\left\{(2 E A P)^{2}(1+j)^{2}-[2 P(1+j) F]^{2}-P^{4}(1+j)^{2}+8 E A(E A\right. \\
\left.+F) P^{2}(1+j)\right\} L_{0}^{4} \\
a_{1}=4 P^{3} F(1+j)^{2} L_{0}^{5} \\
a_{0}=P^{4}(1+j)^{2} L_{0}^{6}
\end{gathered}
$$

Here, as in the case of the nonsliding tensile model [6], a separate equation can be derived for calculation of the final tension, $T$, and the angle of deflection, $\alpha$. Equation (2) gives 


$$
\frac{\sqrt{4 T^{2}-P^{2}}}{2 T}=\cos \alpha
$$

Substituting $\cos \alpha$ from Eq. (26) into Eq. (20) gives the equation for $T$ as

$$
b_{6} T^{6}+b_{5} T^{5}+b_{4} T^{4}+b_{3} T^{3}+b_{2} T^{2}+b_{1} T+b_{0}=0
$$

the coefficients of which are

$$
\begin{gathered}
b_{0}=-4(E A)^{2} P^{2}(F j-E A)^{2}-(E A)^{2}(1+j)^{2} P^{4} \\
b_{1}=-8 E A F P^{2}(1+j)(F j-E A)-8(1+j)(E A)^{2} P^{2}(E A+F) \\
+2 E A(1+j)^{2} P^{4} \\
b_{2}=16(E A)^{2}(F j-E A)^{2}-4(1+j)^{2} F^{2} P^{2}-8(1+j)(E A) P^{2}(E A \\
+F)+8 P^{2}(E A)^{2}(1+j)^{2}-(1+j)^{2} P^{4}-16(E A)^{2}(E A+F)^{2} \\
b_{3}=32 E A F(F j-E A)(1+j)+32(1+j)(E A)^{2}(E A+F) \\
+16 E A(1+j)^{2} P^{2} \\
b_{4}=16(1+j)^{2} F^{2}+32(1+j) E A(E A+F)-16(E A)^{2}(1+j)^{2} \\
+8(1+j)^{2} P^{2} \\
b_{5}=-32 E A(1+j)^{2} \\
b_{6}=-16(1+j)^{2}
\end{gathered}
$$

Equation (21) can be rearranged to give the equation to yield an explicit for direct calculation of $\alpha$. Alternatively the solution for deflection ( $y$ ) from Eq. (25) can be substituted into Eq. (22) (namely, $\alpha=\tan ^{-1}\left(y / L_{0}\right)$ ), to give $\alpha$. Also, once Eq. (27) is solved, Eq. (26) can be rewritten as $\alpha=\cos ^{-1}\left(\sqrt{4 T^{2}-P^{2}} / 2 T\right)$ to readily yield $\alpha$.

\section{Finite Element Simulation of Frictionless Suspen- sion of a Bone Fragment on Pretensioned K-Wires}

Modeling of suspension of the bone segment (as hollow cylinders), the bone on the wires is also unprecedented by finite element analysis (FEA) or otherwise. The free-sliding tensile formulation presented in Sec. 2 can model the actual clinical situation for K-wires used in Ilizarov appliance. Three finite element models were made and analyzed for the configurations shown in Fig. 5-7. Nonlinearity of the behavior of thin wires in Ilizarov appliance is well established $[4,6-9,16,17,19,20]$; thus FE analyses were performed nonlinearly by ABAQUS/STANDARD to check the results obtained from Eq. (25).

$\mathrm{K}$-wires were modeled as identical thin solid cylinders ( $d$ $=1.8 \mathrm{~mm}$ and $L=180 \mathrm{~mm}$ ). The bone segment was modeled as solid hollow cylinder (the internal diameter was $10 \mathrm{~mm}$ and the outside diameter was $15 \mathrm{~mm}$ ). Material properties were defined in terms of Young's modulus and Poisson's ratio; classic values of $E_{s s}=200 \mathrm{GPa}$ and $\nu_{s s}=0.3$ for stainless steel were applied to the wires, and $E_{\text {bone }}=22 \mathrm{GPa}$ and $\nu_{\text {bone }}=0.35$ were used for the hollow cylinder representing the bone $[8,21]$. K-wires passed through the holes of the same diameter in the bone at 90/90 deg as well as $45 / 135$ deg angle to each other. To simulate the assumption that bone can slide freely along the wire, all interactions between the wires and the holes in the bone through which wires were passed were defined tangentially as frictionless and normally as hard contact. Analyses were performed in the absence of any pretension, as well as under all four clinically applied pretensions. The analyses were carried out in two steps.

First, the pretension was applied to one end of each K-wire, while the other end was fully constrained. No other boundary condition was applied to the model at this step, and also no interaction was defined in the model in this step. Bone-wire assembly

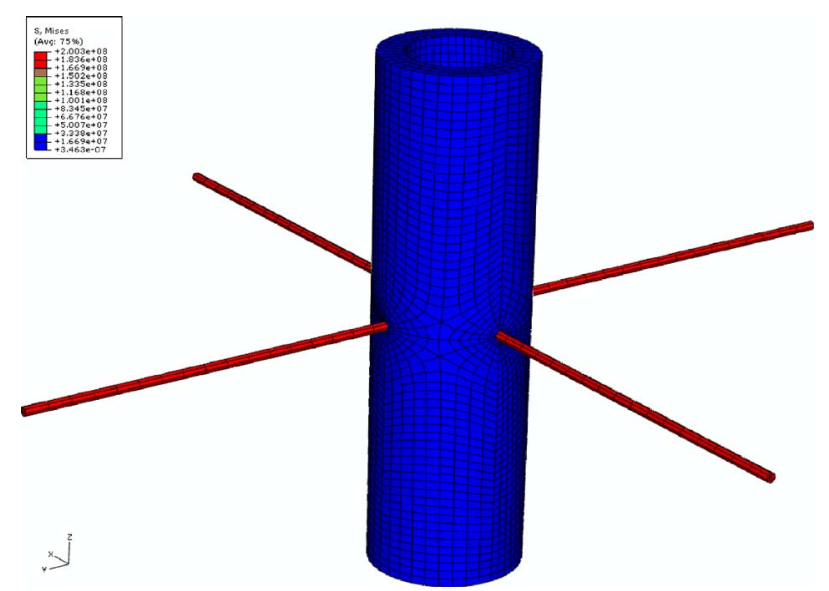

Fig. 4 Pretensioning step for FEA of the wire-bone interaction $(F=490 \mathrm{~N}$, unit of stress is $\mathrm{Pa})$

at the end of this step for a pretension of $F=490 \mathrm{~N}$ is illustrated in Fig. 4, which also shows the stress levels in the model.

In the second, i.e., axial loading of the bone-wire assembly, which means transverse loading of the K-wires, both ends of the $\mathrm{K}$-wires were fully restrained. The load representing the weight of the patient was uniformly applied at the upper end of the cylinder to simulate the axial compression. No boundary condition was applied to the cylinder, and the interaction was defined between the surfaces of K-wires and the cylinder surfaces in contact with the K-wires, which was frictionless tangentially and hard normally. Figure 5 shows the results for stress levels in an assembly with two K-wires with perpendicular axes after both pretensioning and transverse loading of the wires, where $F=490 \mathrm{~N}$ and $P$ $=200 \mathrm{~N}$ per wire. Figure 6 demonstrates the stress contours of a cylinder-wire assembly with two pairs of perpendicular K-wire at 90 deg, while Fig. 7 shows the similar results for an analysis were K-wires were at 45 deg. In assemblies in Figs. 6 and 7, parts other than the cylinder and K-wires are shown, which are display parts and were not included in the analyses. Due to the small diameter of the wires, they should be watched carefully for stress concentration locations. Figure 5 also shows that for the regions in the wire at the immediate vicinity of the clamps or the point of insertion of wire into the bone, the local stresses exceed the yield point of the stainless steel, which is reported to be $520 \mathrm{MPa}$ [21]. Therefore, it can be assumed that plastic deformation is certain to take

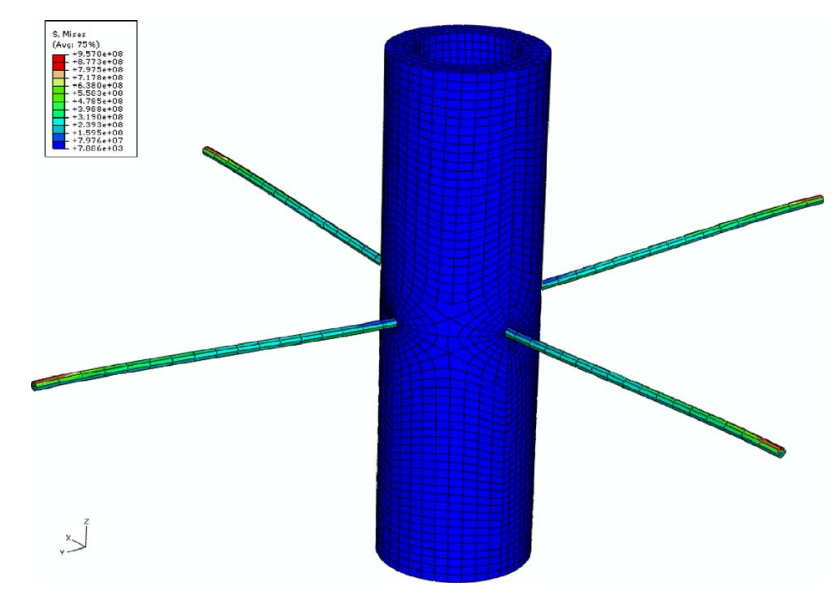

Fig. 5 Application of a transverse load of $P=400 \mathrm{~N}$ on pretensioned wires $(F=490 \mathrm{~N}$, unit of stress is $\mathrm{Pa})$ by applying equivalent pressure to the upper surface of the cylinder (free bone-wire sliding was allowed) 


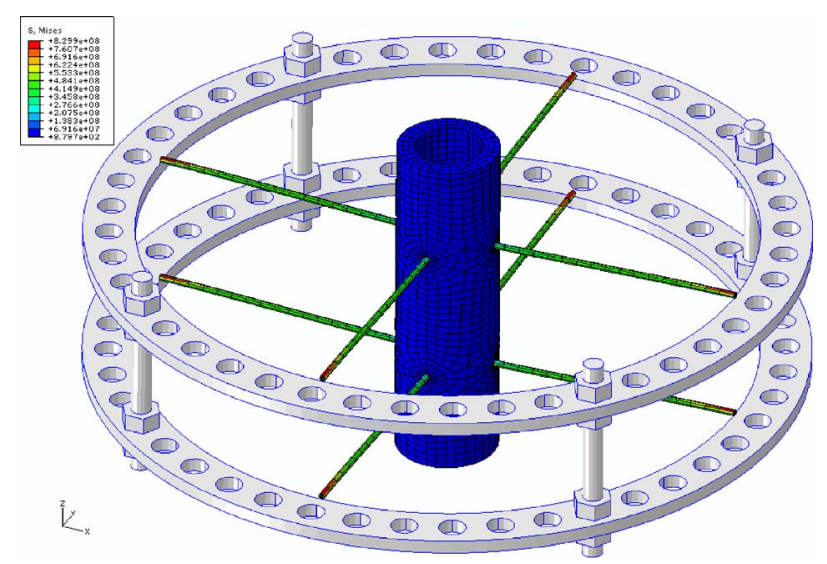

Fig. 6 Stress levels for frictionless suspending of the bone (as a cylinder) on four K-wires at $90 \mathrm{deg}(F=883 \mathrm{~N}, P=300 \mathrm{~N}$, unit of stress is $\mathrm{Pa}$ )

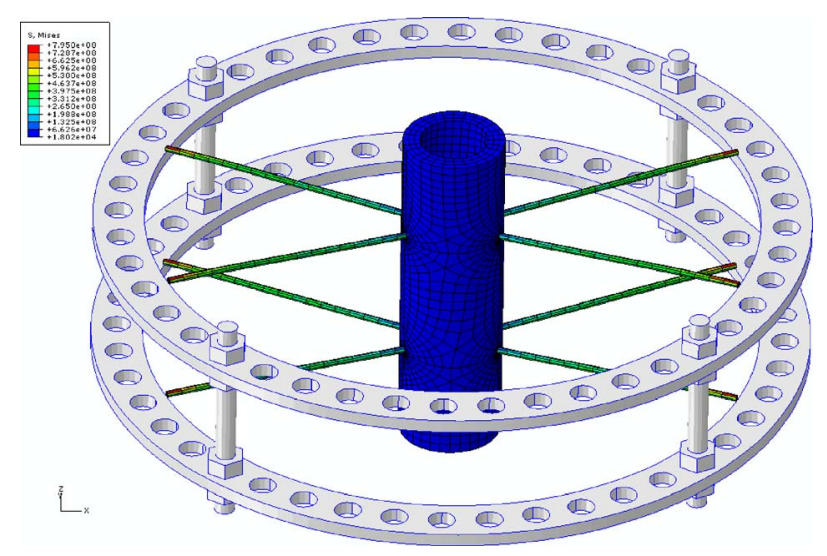

Fig. 7 Stress contours for frictionless suspension of the bone (i.e., cylinder) on four K-wires at $45 \mathrm{deg}(F=490 \mathrm{~N}, P=300 \mathrm{~N}$, unit of stress is $\mathrm{Pa}$ ).

place at those regions. The occurrence of these local yieldings is believed to reinforce the basis of application of a purely elastic tensile model to the wires' behavior, which is applied in the current paper $[6,7,19]$.

\section{Results and Discussions}

The results for deflection versus load from Eq. (25) are plotted in Fig. 8(a) for loads of up to $50 \mathrm{~N}$, at different pretensions as well as in the absence of pretensioning. It shows that if the load is kept within that range, for pretensions of more than $90 \mathrm{kgf}(883 \mathrm{~N})$ the load-deflection behavior is linear. Figure $8(b)$, when compared with Fig. 8(a), shows that as the applied load grows so does the nonlinearity in the K-wire behavior. Figures $8(a)$ and $8(b)$ also show that the nonlinearity decreases as the pretension increases. In Fig. 9, the results for deflection versus load from Eq. (25) as well as the results from no-sliding model [7] are plotted, which shows that the free-sliding model (Eq. (25)) gives higher deflections for the same given parameters. This quantifies the effect of free sliding of bone on the K-wires. Results for stiffness were obtained implicitly from Eq. (25), as tangent modulus, i.e., $K$ $=K_{t}=\partial P / \partial y$, which are plotted in Fig. 10 and proved to be less than those obtained from tensile no-sliding model as given in Ref. [6].

Results for deflections (along the bone axis) from FE analyses for K-wires at 90/90 deg as well as at 45/135 deg are plotted in Fig. 11, which show excellent agreement (almost identical) be-
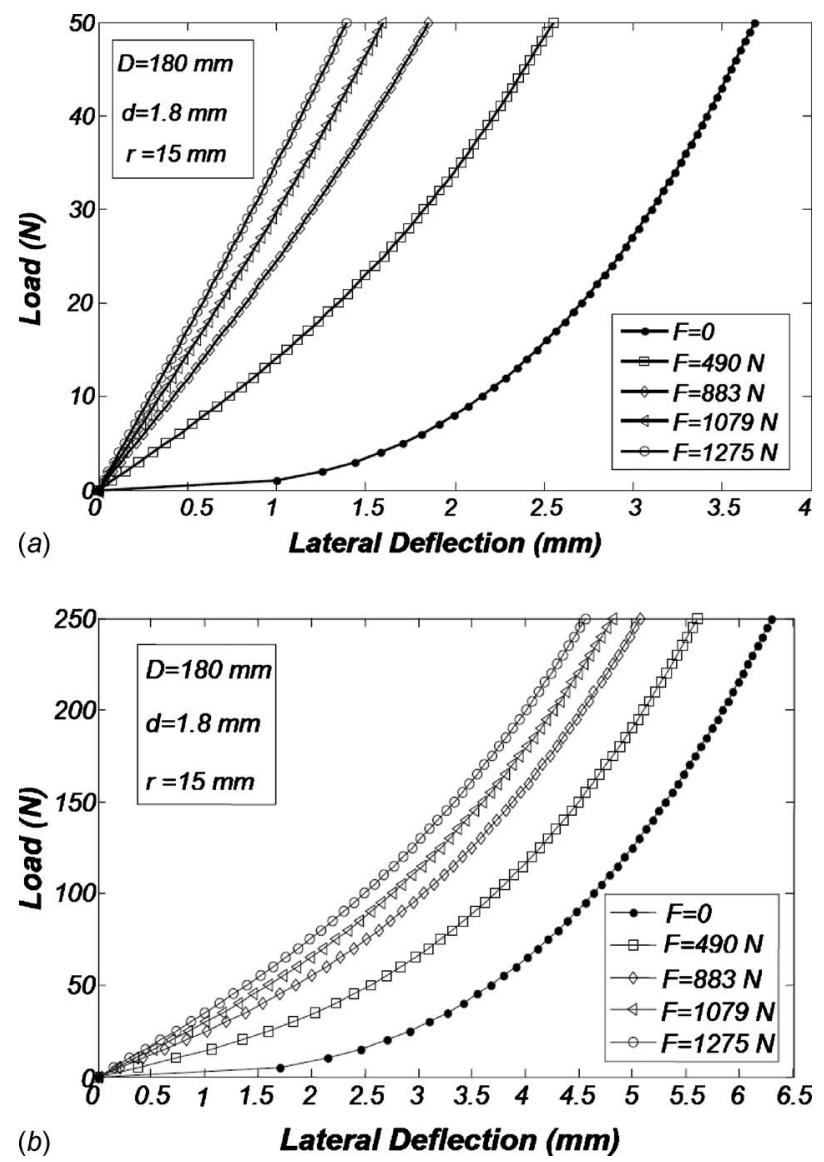

Fig. 8 Load versus deflection curves for a K-wire under different pretensions (tensile free-sliding model), for (a) a small range and (b) an extended range of transverse loads

tween the two set of results for all the clinically applied pretensions, as well as in the absence of pretensioning. This shows that the $\mathrm{K}$-wire behavior is not dependent on the angle between them, even in the case of free sliding of the bone on the K-wires, which means that the reports of effect of wire angles on the loaddeflection behavior of the fixator (in axial compression) [9] must be due to the ring deformation. This requires further investigation on the behavior of the rings in all circular (ring) fixators. Figure 11 also shows that the nonlinearity is increased as the pretension decreases, which confirms the trend observed in the results from analytical solution plotted in Fig. 8.

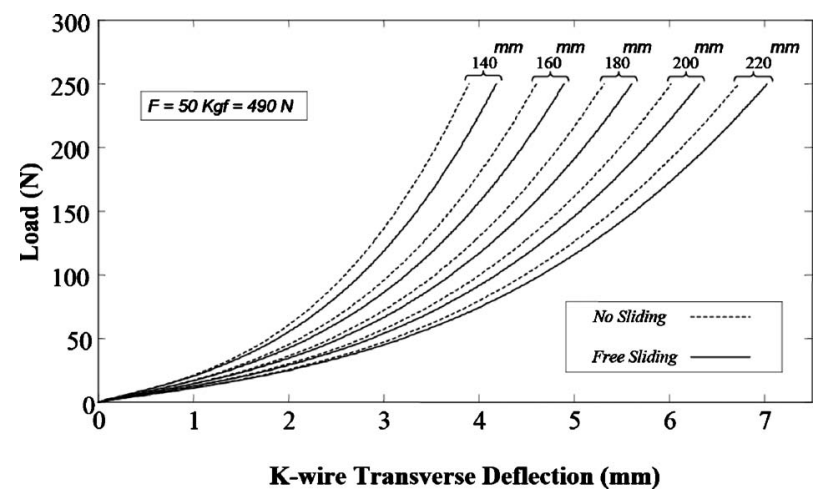

Fig. 9 Comparison of load versus deflection curves for a wire under a given pretension with different wire spans modeled by the two tensile models 


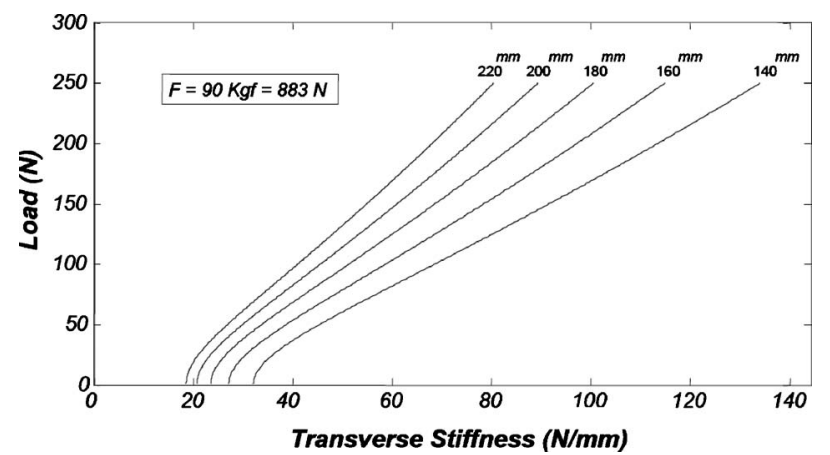

Fig. 10 Load versus stiffness curves for a wire under a given pretension for different wire spans (tensile free-sliding model), where stiffness is defined as tangent modulus (i.e., $K=K_{t}$ $=\partial P / \partial y)$

Figures $12-15$ offer comparisons between the results for deflection versus load at different pretensions from Eq. (25) and those from FE analyses. They show good agreement between the two sets of results, which confirms the applicability of the analytical solution. The effect, which can be called the linearizing effect of

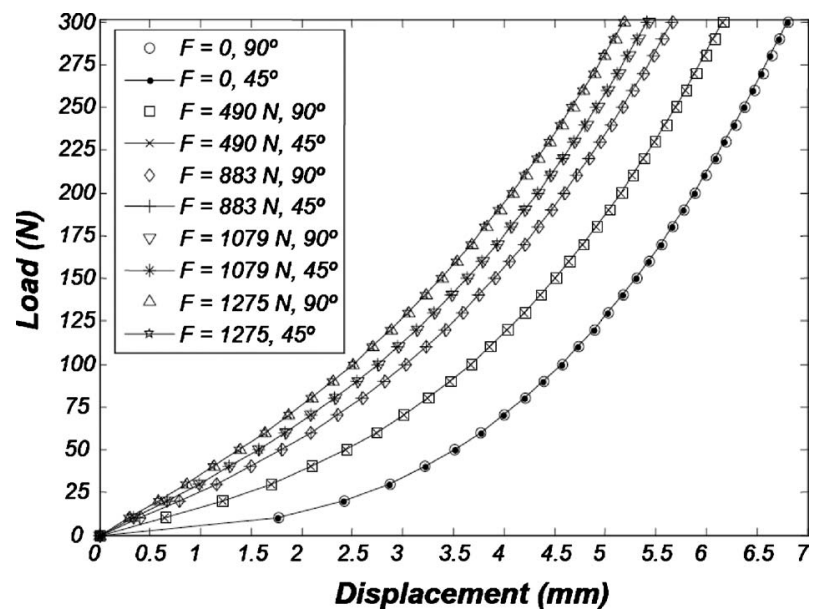

Fig. 11 Load versus deflection curves from FE analysis of two different configurations for K-wires angles (90 deg and $45 \mathrm{deg}$, as seen in Figs. 6 and 7, respectively)

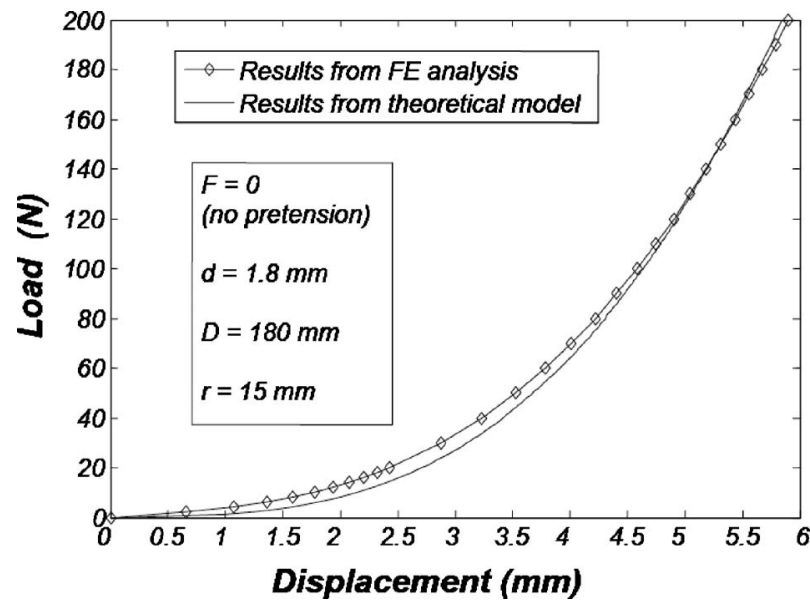

Fig. 12 Results for load versus deflection from FEA for a pair of K-wires subjected to lateral compression by the bone (see Fig. 5), compared with analytical solution from Eq. (25), in the absence of friction and pretension

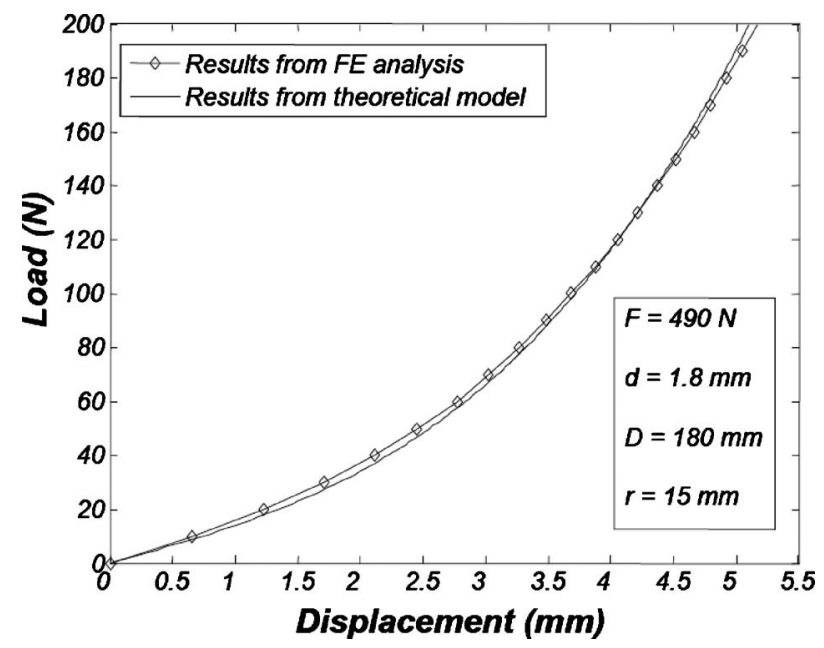

Fig. 13 Results for load versus deflection from FEA for a pair of K-wires subjected to frictionless lateral compression by the bone (see Fig. 5), compared with analytical solution from Eq. (25), at a relatively low clinical pretension $(F=490 N)$
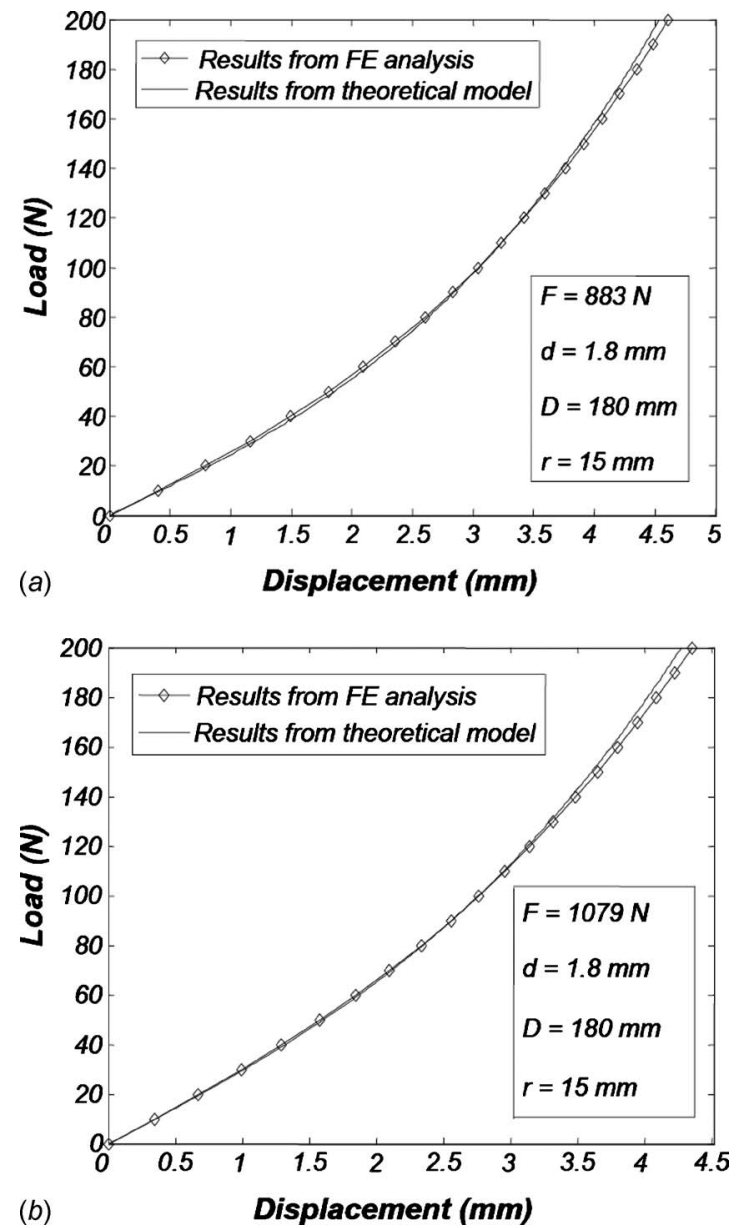

Fig. 14 Results for load versus deflection from FEA for a pair of wires subjected to frictionless lateral compression by the bone (see Fig. 5), compared with analytical solution from Eq. (25), at medium range clinical pretensions: (a) $F=883 \mathrm{~N}$ and $($ b) $F=1079 \mathrm{~N}$ 


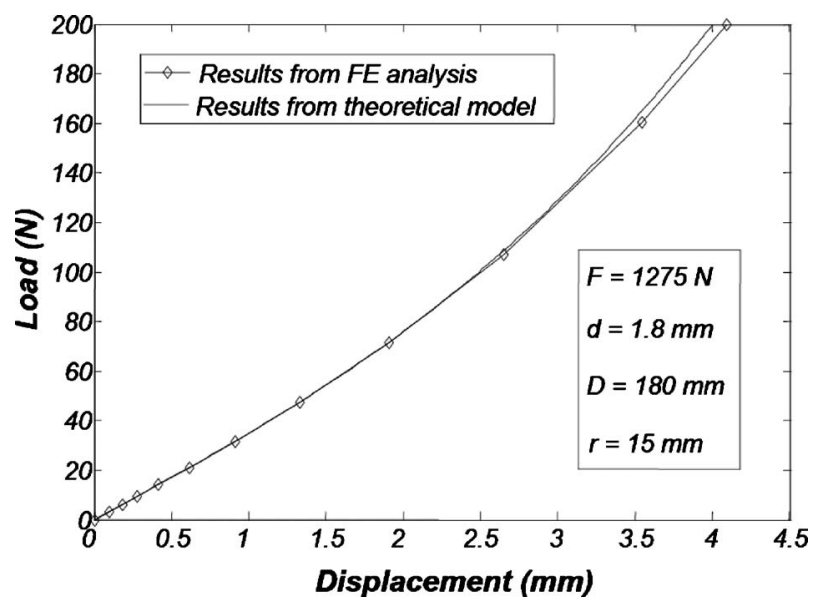

Fig. 15 Results for load versus deflection from FEA for a pair of wires subjected to transverse compression by the bone (see Fig. 5), compared with analytical solution from Eq. (25). At a high clinical pretension $(F=1275 \mathrm{~N})$, the bone-wire interaction assumed to be frictionless.

pretension, has been also observed when comparing these figures. As the material was assumed to obey Hooke's law, the nonlinearity observed in the results from both the theoretical and FE analyses, which are plotted in Figs. 8-15, ought to be geometric. The "roots" command in the MATLAB software has been used to solve Eqs. (25) and (27). Results from Eq. (27) for final tension in the wire are plotted in Fig. 16 at different clinical pretension.

Tension in the K-wires as predicted by Eq. (25) for a relatively small ring diameter $(D=140 \mathrm{~mm})$, as well as for a larger ring $(D=220 \mathrm{~mm})$, are plotted in Fig. 17, for both low and high pretension levels. It shows the small effect that the length can have on the final tension in a K-wire, which is even smaller at the high pretension of $1275 \mathrm{~N}$. It also could be explained by Eq. (27), which contains no explicit term of length of wire (e.g., $L_{-2}, L_{-1}$, and $L$ ). Nevertheless, Eq. (27) does include terms involving $j$, which means that the ratio of the bone diameter to the wire length (i.e., ring diameter) can affect the ultimate (final) tension developed in the wire due to the application of lateral load $(P)$. Results for angle of deflection ( $\alpha$ in Fig. 2), for a small ring diameter, is plotted in Fig. 18(a) for a high and a relatively low pretension. The same graphs for a large ring are plotted in Fig. 18(b). Both of

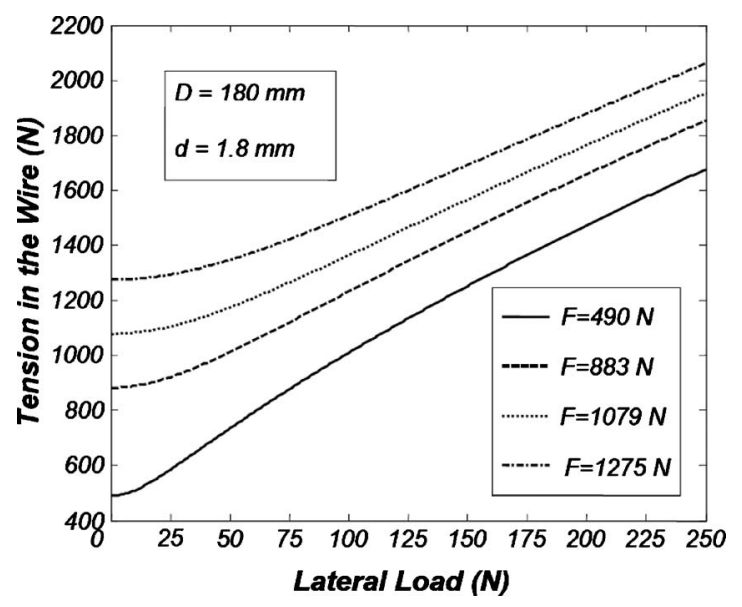

Fig. 16 Final tension in a K-wire subjected to frictionless transverse (lateral) compression by a cylinder, as predicted by analytical solution in Eq. (27), at different clinically applied pretensions
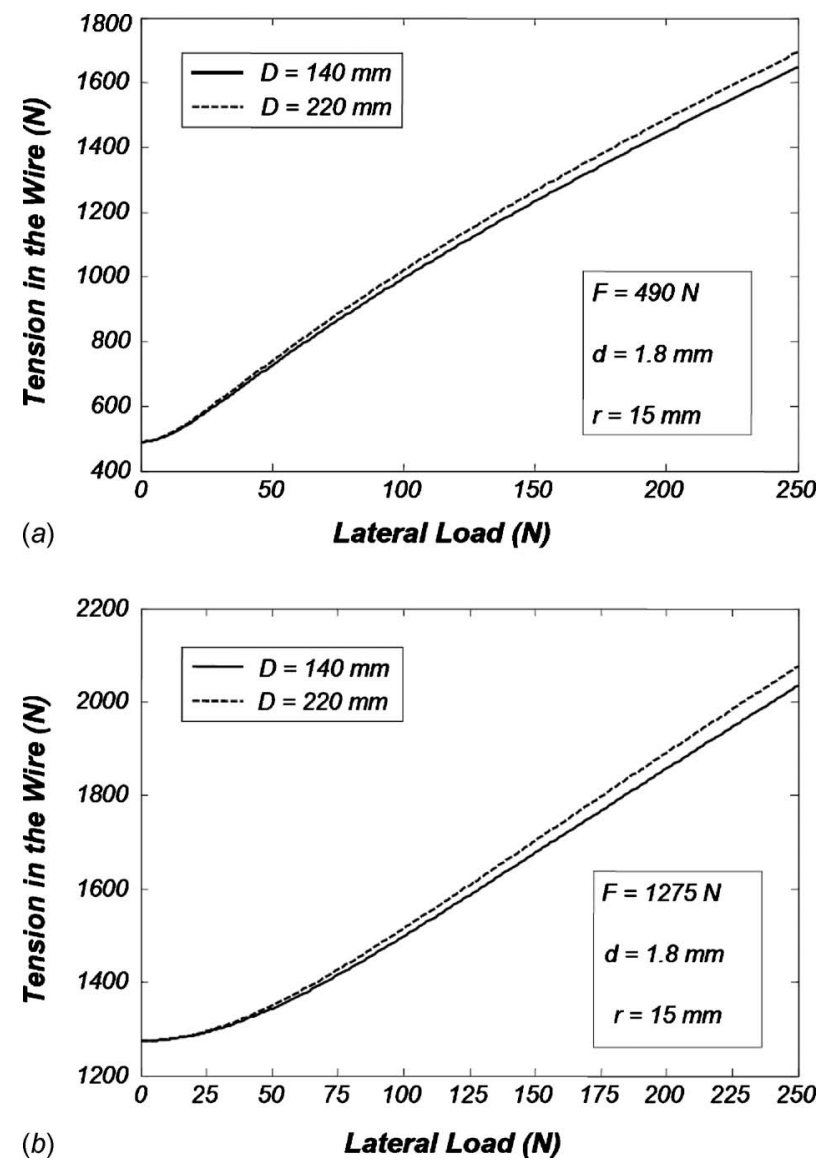

Fig. 17 Effect of length on the final tension in the K-wires subjected to frictionless lateral compression by a cylinder, as predicted by analytical solution in Eq. (27), at (a) a low clinical pretension and $(b)$ a high pretension

these figures show that the effect of the ring size on the angle of deflection is small, which decreases as the pretension increases.

Comparing Eq. (25) and Eq. (27) with the equations for maximum deflection and final tension in the wires in Ref. [7], respectively, shows how the solutions have grown in complexity by introducing the effect of sliding between bone and wires. Not only the coefficients have more terms but also the degree of the equation is raised from 4 to 6 . The fact that polynomials of up to fourth degree can be solved using radicals (root extractions) implies that the same solution technique is also rendered inapplicable. Nonetheless, Eqs. (25) and (27) can be solved numerically, which of course is easily carried out by a variety of methods or simply by a single command in a number of technical computing software packages (e.g., roots ([...]) in MATLAB). It should also be mentioned that although generally there are a set of six roots for a sixth degree polynomial equation, in the case of Eqs. (25) and (27), it was easy to distinguish the sole acceptable solution from other unacceptable roots, all of which were either complex, negative, or abnormally large or small values. They offer improvements to the equations in Ref. [7]. The equations for deflection and final tension in the wire presented in Ref. [7] merely constitute special cases of Eqs. (25) and (27) in which $r=j=0$.

In Fig. 19, curves for stiffness versus tension in the wire are plotted, which are a set of parallel straight lines. It shows that stiffness defined as tangent modulus is linearly dependent on the tension in the wire. This stiffness-tension proportionality underlines the significance of the pretensioning, without which the wire would lack any initial stiffness. Figures $8(a)$ and $8(b)$ can be compared with the graphs from experimental data presented in Refs. $[16,4]$, respectively. To make the comparison clearer, Fig. 20 was 

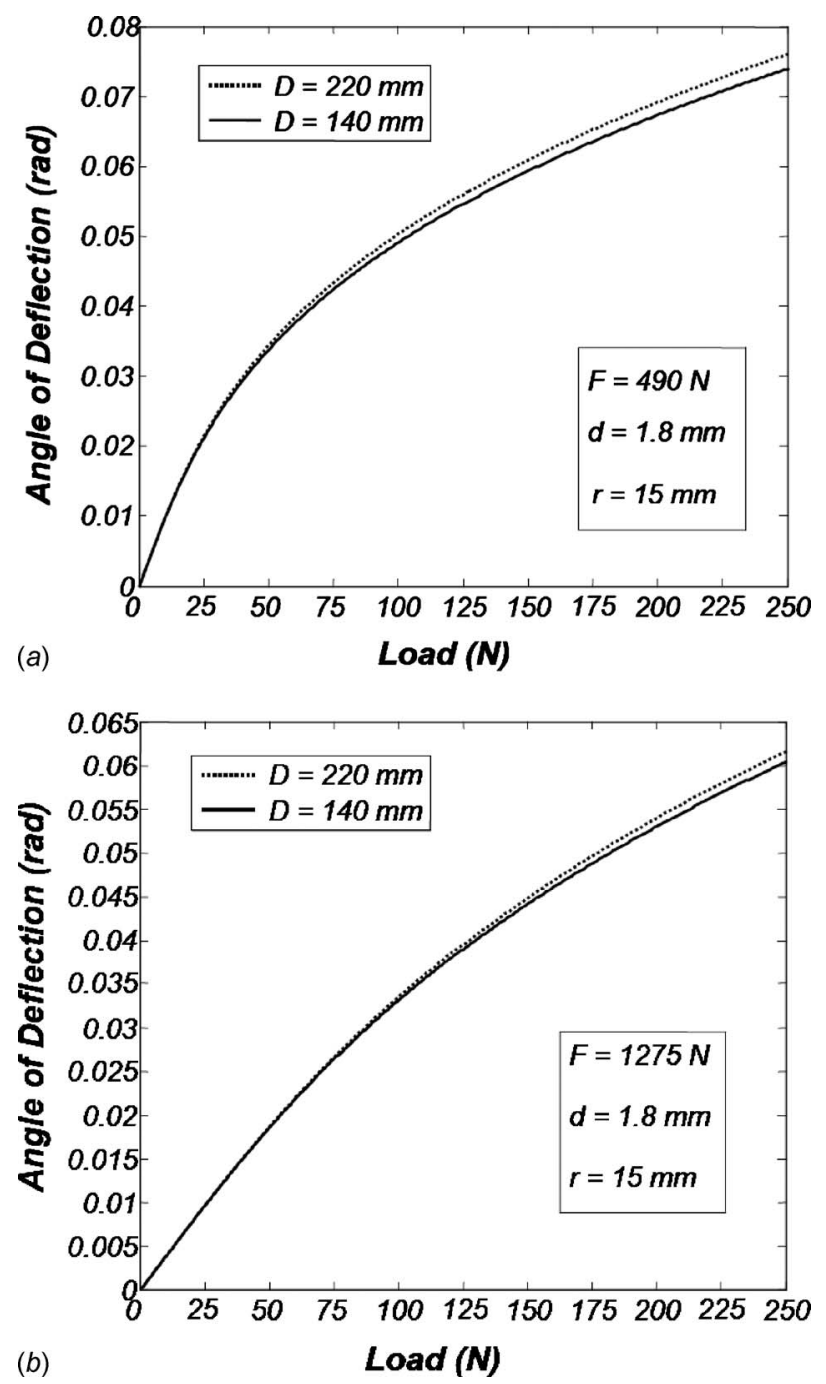

Fig. 18 Effect of length on the angle of deflection of the $\mathrm{K}$-wires subjected to frictionless lateral compression by a cylinder, as predicted by analytical solution in Eq. (25), at (a) a low clinical pretension and $(b)$ a high pretension

produced in which the graph produced from Eq. (25) is shown alongside the graphs from experimental data, and the FE analysis published by Watson et al. [16]. It shows that the results obtained from Eq. (25) agree to the published experimental data.

\section{Conclusions}

Taking into account the wire segment that is placed inside the bone increased the lateral deflection of the wire and thus reduced the stiffness. The results from the obtained polynomial equation showed good agreement with the results yielded by the FE analyses. However, this should not be construed as validation of either method against the other. Having a single polynomial equation for the nonlinear deflection of the wire simplifies the solution relative to the FE method, which needs to be solved nonlinearly. In this work, wires were modeled using solid elements in contrast to the beam elements, for the model to be able to accommodate the bone-wire slippage in the model, which was carried out via defining contact between bone and wire. Beam elements have been previously used for modeling the wires in circular fixators [8-21], where they are transfixed through the bone segment, which means that the interaction was not defined at the bone-wire interface to allow the reported wire-bone slippage (or sliding) [4,5,9]. The results from theoretical solution showed that length of the wire

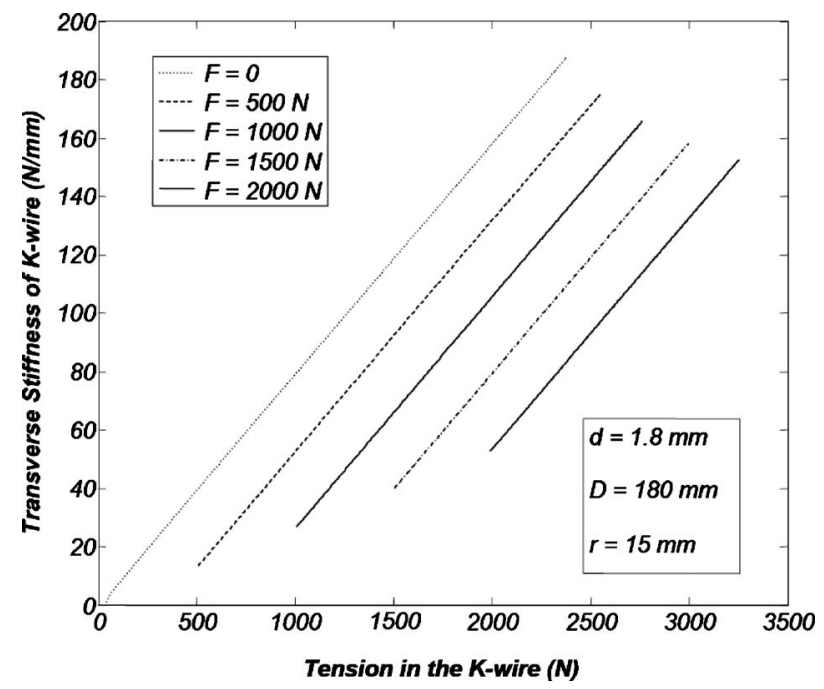

Fig. 19 K-wire stiffness defined as tangent modulus versus tension in the wire under different pretensions and transverse loads from $\mathrm{ON}$ to $500 \mathrm{~N}$ for a $180 \mathrm{~mm}$ diameter ring

has only a small effect on the tension developed in the wire due to transverse loading and also on the angle by which the wire is deflected. They also demonstrated that the stiffness of a wire is proportional to its tension. As expected, changing the wire orientation from $90 / 90$ deg to $45 / 135$ deg did not change the results yielded by the FE analyses. It implies that the reported change in the stiffness of fixator for different wire angles [9] must be from deformation of the rings or other factors. As shown in Fig. 1, the rings are supported by interconnecting rods, which connect the rings and can be assumed to be strong enough to allow their deformation to be ignored in the analysis of the fixator assembly. Nonetheless, the rings will deform as curved beams loaded by the wires (see Fig. 2), with boundary condition applied where the interconnecting rods are placed on the ring. Magnitude of the load applied to the ring is obviously the same as the tension in the wire (and of course in opposite direction), which is predicted by Eq. (27) and illustrated in Figs. 16 and 17. They show that tension is relatively high and thus is capable of inducing deflections in the ring between two adjacent interconnecting rods, which can affect the overall stiffness of the fixator.

In the end, it should be emphasized that the scope of this paper is limited to the behavior of the slender Kirschner wires as used as

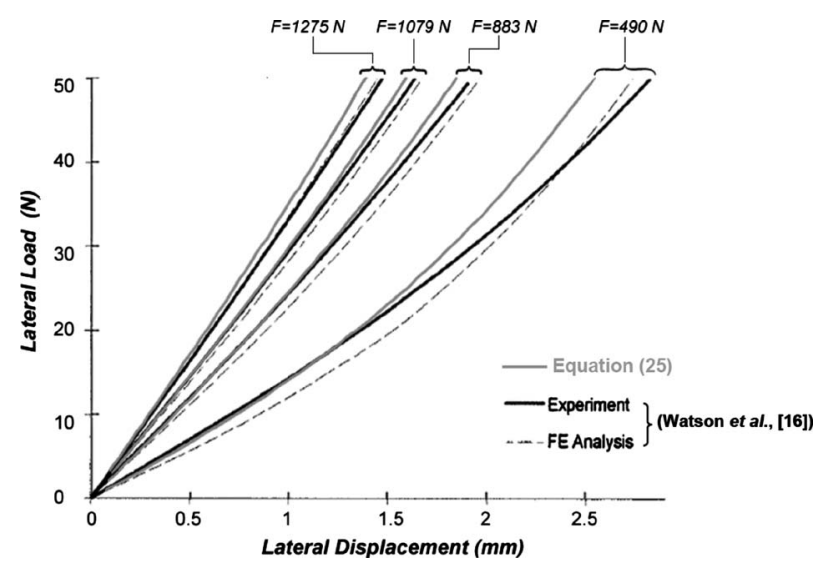

Fig. 20 A comparison of Load versus deflection curves for a K-wire under different pretensions (in a small range of applied lateral loads) obtained from Eq. (25), and experimental data published by Watson et al. [16] 
"a part" of circular external fixators. Here, including the bone in the model was solely aimed at accommodating bone-wire interaction in the FE analysis. The need for a comprehensive modeling of the entire geometry of the bone-fixator assembly, e.g., via FE technique, still persists. Such a model should be geometrically more accurate and account for interaction between different components, allowing more complicated loadings, i.e., tension/ compression, torsion, bending, or combinations of them, to be simulated. In this paper, the interaction between the bone and the wires were modeled as frictionless, which as mentioned above only reflects an ideal behavior and will necessitate further simulations to be carried out with, perhaps, different friction coefficients. Material properties of the bone is another issue to be addressed in further FE studies, such as bone creep behavior as a result of it being loaded dynamically over relatively long periods of time.

\section{Notations and Nomenclature}

$\alpha=$ angle of deflection of the wire, see Fig. 2

$\nu=$ Poisson's ratio

$A=$ cross sectional area of the K-wire (or beam)

$a_{0}, a_{1}, \ldots, a_{6}=$ coefficients in Eq. (25)

$b_{0}, b_{1}, \ldots, b_{6}=$ coefficients in Eq. (27)

$D=$ internal diameter of the ring (in circular fixators)

$d=$ diameter of K-wire (or beam)

$E=$ Young's modulus of elasticity of the material

$F=$ pretension (pretensile force) applied longitudinally to K-wire or beam

$j=$ ratio of outer radius of the bone at the point of insertion of the $\mathrm{K}$-wire, to the bone-clamp distance $\left(j=r / L_{0}\right)$

$K=$ transverse stiffness of the wire (here $K=K_{t}$ $=\partial P / \partial y)$

$L=$ length of the wire segment connecting bone to clamp after both pretensioning and lateral loading, see Fig. 3(b)

$L_{0}=$ bone-clamp distance (i.e., length of the wire segment connecting bone to clamp after pretension)

$L_{-1}=$ untensioned length of the wire segment which is stretched due to its final tension (i.e., $T$ ) to cover the bone clamp distance (i.e., $L_{-1} \stackrel{T}{\rightarrow} L$ )

$L_{-2}=$ untensioned length of the wire segment which is stretched due to pretensioning to cover the

bone-clamp distance (i.e., $L_{-2} \rightarrow L_{0}$ )

$P=$ load applied transversely to the K-wire

$r=$ outer radius of the bone (or the cylinder) at the point of wire insertion, see Fig. 2

$r_{0}=$ half the untensioned length of the wire segment which is stretched due to pretension to cover the (outer) diameter of the bone (i.e., $\stackrel{F}{\left.r_{0} \rightarrow r\right)}$

$r_{-1}=$ half the untensioned length of the wire segment which is stretched due to its final tension (i.e., $\left.T_{r}\right)$ to cover the diameter of the bone $\left(r_{-1}\right.$ $T_{r}$

$T=$ final tension in the K-wire or beam (after both pretensioning and transverse loading) in the bone-clamp segment

$T_{r}=$ final tension in the wire segment under the bone

$y=$ maximum transverse deflection of the wire (see Fig. 2)

\section{References}

[1] Solomin, L. N., 2008, The Basic Principles of External Fixation Using the Ilizarov Device, Springer-Verlag, Italy.

[2] Aronson, J., 1997, "Current Concepts Review—Limb-Lengthening, Skeletal Reconstruction, and Bone Transport With the Ilizarov Method," J. Bone Jt. Surg., Am. Vol., 79, pp. 1243-58.

[3] Catangi, M. A., Malzev. V., and Kirienko, A., 1994, Advances in Ilizarov Apparatus Assembly, Medicalplastic srl, Milan, Italy, pp. 3-8.

[4] Watson, M. A., Mathias, K. J., and Maffulli, N., 2000, "External Ring Fixators: An Overview," Proc. Inst. Mech. Eng., Part H: J. Eng. Med., 214, pp. 459-470.

[5] Kummer, F. J., 1992, "Biomechanics of the Ilizarov External Fixator," Clin. Orthop. Relat. Res., 280, pp. 11-14.

[6] Zamani, A. R., and Oyadiji, S. O., 2008, "Analytical Modelling of Kirschner Wires in Ilizarov Circular External Fixators Using a Tensile Model," Proc. Inst. Mech. Eng., Part H: J. Eng. Med., 222, pp. 967-976.

[7] Zamani, A. R., and Oyadiji, S. O., 2009, "Analytical Modelling of Kirschner Wires in Ilizarov Circular External Fixators as Pretensioned Slender Beams," J. R. Soc., Interface, 6(32), pp. 243-256.

[8] Zhang, G., 2004, "Avoiding Material Nonlinearity in an External Fixation Device," Clin. Biomech. (Bristol, Avon), 19, pp. 746-750.

[9] Bronson, D. G., Samchukov, M. L., Birch, J. G., Browne, R. H., and Ashman, R. B., 1998, "Stability of External Circular Fixation: A Multivariable Biomechanical Analysis," Clin. Biomech. (Bristol, Avon), 13, pp. 441-448.

[10] Kristiansen, T., Fleming, B., Neale, G., Reinecke, S., and Hope, M. S., 1987, "Comparative Study of Fracture Gap Motion in External Fixation," Clin. Biomech. (Bristol, Avon), 2(4), pp. 191-195.

[11] Fleming, B., Paley, D., Kristiansen, T., and Pope, M., 1989, "A Biomechanical Analysis of the Ilizarov External Fixator," Clin. Orthop. Relat. Res., 241, pp. $95-105$.

[12] Podolsky, A., and Chao, E. Y. S., 1993, "Mechanical Performance of Ilizarov Circular External Fixators in Comparison With Other External Fixators," Clin. Orthop. Relat. Res., 293, pp. 61-70.

[13] Chao, E. D. Y. S., Aro, H. T., Lewallen, D. G., and Kelly, P. J., 1989, "The Effect of Rigidity on Fracture Healing in External Fixation," Clin. Orthop. Relat. Res., 241, pp. 24-35.

[14] Board, T. N., Yang, L., and Saleh, M., 2007, "Why Fine-Wire Fixators Work: An Analysis of Pressure Distribution at the Wire-Bone Interface," J. Biomech., 40, pp. $20-25$.

[15] Watson, M. A., Mathias, K. J., Maffulli, N., and Hukins, D. W. L., 2003, "The Effect of Clamping a Tensioned Wire: Implications for the Ilizarov External Fixation System," Proc. Inst. Mech. Eng., Part H: J. Eng. Med., 217, pp. 91-98.

[16] Watson, M. A., Matthias, K. J., Maffulli, N., and Hukins, D. W. L., 2003, "Yielding of the Clamped-Wire System in the Ilizarov External Fixator," Proc. Inst. Mech. Eng., Part H: J. Eng. Med., 217, pp. 367-374.

[17] Hillard, P. J., Harrison, A. J., and Atkins, R. M., 1998, "The Yielding of Tensioned Fine Wires in the Ilizarov Frame," Proc. Inst. Mech. Eng., Part H: J. Eng. Med., 212, pp. 37-47.

[18] Renard, A. J. S., Schutte, B. G., Verdonschot, A., and van Kampen, A. "The Ilizarov External Fixator: What Remains of the Wire Pretension After Dynamic Loading?," Clin. Biomech. (Bristol, Avon), 20, pp. 1126-1130 2005.

[19] Nikonovas, A., and Harrison, A. J. L., 2005, "A Simple Way to Model the Wires in Ilizarov Wires Used in Ring Fixators: Analysis of the Wire Stiffness Effect on Overall Fixator Stiffness," Proc. Inst. Mech. Eng., Part H: J. Eng. Med., 219, pp. 31-42.

[20] Aronson, J., and Harp, J. H., Jr., 1992, "Mechanical Considerations in Using Tensioned Wires in a Transosseous External Fixation System," Clin. Orthop. Relat. Res., 280, pp. 23-29.

[21] Zhang, G., 2004, "Geometric and Material Nonlinearity in Tensioned Wires of an External Fixator," Clin. Biomech. (Bristol, Avon), 19, pp. 513-518. 